\title{
Integrated Production-Distribution Scheduling Problem with Multiple Independent Manufacturers
}

\author{
Jianhong Hao, ${ }^{1}$ Lisi Cao, ${ }^{2,3}$ and Dakui Jiang ${ }^{2}$ \\ ${ }^{1}$ Automotive Engineering Research Institute, China Automotive Technology \& Research Center, Tianjin 300300, China \\ ${ }^{2}$ College of Management and Economics, Tianjin University, Tianjin 300072, China \\ ${ }^{3}$ School of International Business, Tianjin Foreign Studies University, Tianjin 300270, China
}

Correspondence should be addressed to Dakui Jiang; jiangdakui@tju.edu.cn

Received 11 February 2015; Accepted 16 March 2015

Academic Editor: Tadeusz Kaczorek

Copyright (C) 2015 Jianhong Hao et al. This is an open access article distributed under the Creative Commons Attribution License, which permits unrestricted use, distribution, and reproduction in any medium, provided the original work is properly cited.

\begin{abstract}
We consider the nonstandard parts supply chain with a public service platform for machinery integration in China. The platform assigns orders placed by a machinery enterprise to multiple independent manufacturers who produce nonstandard parts and makes production schedule and batch delivery schedule for each manufacturer in a coordinate manner. Each manufacturer has only one plant with parallel machines and is located at a location far away from other manufacturers. Orders are first processed at the plants and then directly shipped from the plants to the enterprise in order to be finished before a given deadline. We study the above integrated production-distribution scheduling problem with multiple manufacturers to maximize a weight sum of the profit of each manufacturer under the constraints that all orders are finished before the deadline and the profit of each manufacturer is not negative. According to the optimal condition analysis, we formulate the problem as a mixed integer programming model and use CPLEX to solve it.
\end{abstract}

\section{Introduction}

Machinery and equipment manufacturing play increasingly important role in economic development of China especially in recent years. Meanwhile, many enterprises in China which produce machinery commonly encountered the problem that they need to search for manufacturers who produce nonstandard parts of the machinery, evaluate their production capability, and monitor their operations to ensure the progress and quality for the assigned orders for the reason that most of nonstandard parts manufacturers in China are extremely small and lacking enterprise credits. Therefore, as is shown in Figure 1, some public service platforms are established by third-party organizations to receive orders from machinery manufacturing enterprises, assign orders to the manufacturers which are authenticated by the platform, make schedules for each manufacturer because of its weak operation capabilities, and monitor progress of the production and distribution for each order in each manufacturer. In this paper, we consider the schedule making service of public platform. The service is to schedule production and distribution operations of each manufacturer in a coordinated and efficient manner so as to finish the orders placed by an enterprise before the deadline and make profit for each manufacturer.

In this paper, we consider a simplified version of the integrated production-distribution scheduling problem with multiple independent manufacturers located at different locations for the above-described supply chain. In this problem, the platform receives a set of orders from the enterprise and needs to determine which orders to be assigned to which manufacturers, how to schedule the production of the assigned orders at each manufacturer, and how to schedule the distribution of the completed orders from each manufacturer to the enterprise, so as to optimize the total weight sum of each manufacturer's profit under the constraints that the orders are finished before a deadline and the profit of each manufacturer is not negative.

The problem can be grouped into the integrated production and outbound distribution scheduling problems [1] with multiple plants at different locations. As pointed out by 


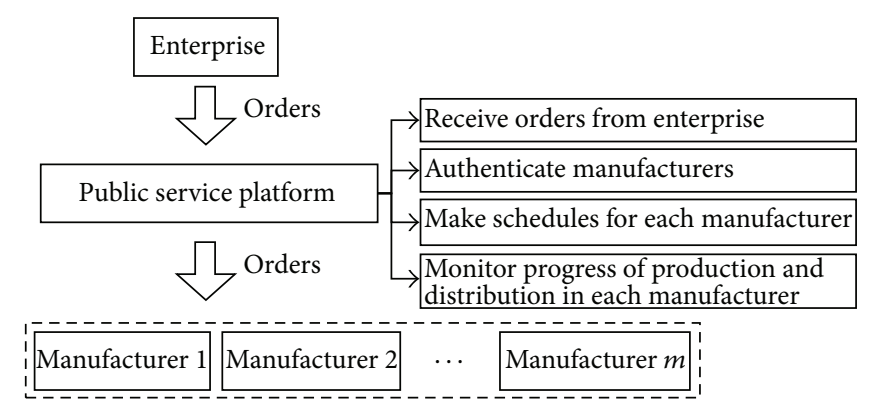

FIGURE 1: Supply chain with a public service platform.

Chen [1] in 2010, "the research on integrated productiondistribution models at the detailed scheduling level is fairly recent; the majority of the work in this area was done in the last five years." In recent integrated production-distribution models, the objective of which is to optimize one or a combination of the time-based and cost-based performance measures, Hall and Potts [2,3] considered a different set of models with batch delivery by direct shipping method that treats both delivery lead time and transportation cost as part of the objective in the supply chain of one plant and several customers. Pundoor and Chen [4] considered maximum delivery tardiness and the total distribution cost in the models. Chen and Vairaktarakis [5] studied a set of models with batch delivery by routing method that treats mean lead time and maximum lead time as part of the objective, respectively. Chen and Pundoor [6] also introduced a model of one supplier and one customer, the objective of which is to make the total distribution cost minimized subject to the constraint that orders should be finished before their deadlines or average delivery lead time of the orders should be satisfied within a given threshold. Dong et al. [7] proposed an approximation algorithm for a model with single plant and single customer to minimize the delivery time of the last order. In this model, a two-machine open shop is applied to process orders and a single vehicle is used to deliver orders. Ullrich [8] proposed a genetic algorithm for the model with a single plant and multiple customers so as to minimize total tardiness. After that, Low et al. [9] studied the case with heterogeneous fleet of vehicles. However, compared with models with single plant, there are little literatures concentrated on multiple plants. Chen and Pundoor [10] introduced the order assignment and scheduling problem with multiple overseas plants and a domestic distribution center in a global supply chain, and they considered four different performance measures, all of which take into account both delivery lead time and the total cost. Jiang and Li [11-13] expended a model introduced in [10] from one customer to multiple and from one machine in each plant to parallel machines, respectively. Till now, little attention in literatures is paid to the profit as the objective of the model because the plants in the supply chain belong to the same manufacturer and the profit can be calculated easily by the price of orders, production cost, and transportation cost.

The structure of the supply chain with a public service platform is similar to that of manufacturing collaborative alliance [14] which plays a role in making independent manufacturing enterprises cooperate to finish a task that one of the enterprises cannot undertake. However, most of researches on manufacturing collaborative alliance are concentrated on supplier selection and order allocation stages (e.g., [15-17]; among others). In this paper, we will not only consider order assignment but also take scheduling in production stage and distribution stage into consider.

The objective of this paper is to describe the problem mathematically in a coordinate manner and analyse optimal conditions of the problem to simplify the problem and solve the problem.

\section{Formulation}

2.1. Problem Description. A public service platform receives $n$ independent orders from an enterprise, $N=\{1,2, \ldots, n\}$, at time 0 , and all of the orders should be processed and delivered to the enterprise before a deadline $D$. The price of order $j$ afforded by the enterprise is $e_{j}, j \in N$. In the production stage, there are $m$ manufacturers authenticated by the platform, $M=\{1,2, \ldots, m\}$, and manufacturer $i$ has $r_{i}$ parallel machines and is capable of producing all the orders, $i \in M$. It takes $p_{i j}$ units of processing time and $c_{i j}$ units of production cost for manufacturer $i$ to process order $j$, for $i \epsilon$ $M$ and $j \in N$. Each order only needs to be processed by one machine once without interruption. In the distribution stage, we adopt the assumption provided by Chen and Pundoor [10] that completed orders are delivered to the enterprise directly from the manufacturer and no vehicle routing is allowed between any two manufacturers. The assumption makes sense in many practical situations where the manufacturers are far away from each other. The delivery time and delivery cost of a shipment from manufacturer $i$ to the enterprise are $t_{i}$ and $f_{i}$, respectively. Each delivery shipment has a capacity limit; it can carry up to $b$ orders. We assume that each order takes up the same amount of capacity of a shipment and that partial delivery of an order is not allowed. The problem is to assign each order to a manufacturer, schedule the processing of the orders assigned to each manufacturer, and schedule the delivery of the completed orders from each manufacturer to the enterprise, so as to maximize a weight sum of the profit of each manufacturer under the condition that the profit of each manufacturer is not negative.

A solution of the problem includes three parts: order assignment schedule, production schedule, and batch delivery schedule. The order assignment schedule is to determine which orders to be assigned to each machine of manufacturers. The production schedule is to determine which sequence of the orders to use in each machine and when to start processing each order. Similarly, the batch delivery schedule is to determine how many shipments to use from each manufacturer to the enterprise, which orders to be delivered in each shipment, and when each shipment should depart from the manufacturer. For ease of presentation, we use $(\delta, \sigma, \varphi)$ to represent a solution of the problem. $\delta$ describes an order assignment schedule, $\sigma$ describes a production schedule associated with $\delta$, and $\varphi$ describes a batch delivery 
schedule associated with $\sigma$. Denote $\Omega$ as a set including all solutions feasible for the problem.

For a given solution $(\delta, \sigma, \varphi)$, we define the following:

$D_{\max }^{i}(\delta, \sigma, \varphi)$ : the maximum lead time of the orders processing in manufacturer $i$;

$P_{i}(\delta, \sigma, \varphi)$ : the profit of manufacturer $i$.

The problem can be formulated as

$$
\begin{array}{ll}
\operatorname{Max} & \sum_{i \in M} \alpha_{i} P_{i}(\delta, \sigma, \varphi) \\
\text { s.t. } & P_{i}(\delta, \sigma, \varphi) \geq 0, \quad \forall i \in M \\
& D_{\max }^{i}(\delta, \sigma, \varphi) \leq D, \quad \forall i \in M \\
& (\delta, \sigma, \varphi) \in \Omega .
\end{array}
$$

The objective function is to maximize a weight sum of the profit of each manufacturer, where $\alpha_{i}$ is a given constant representing the decision maker's relative preference on manufacturer $i$, which may concern the grade of the manufacturer $i$ evaluated by the authenticate manufacturers service of the platform. The higher is the grade of the manufacturer $i$, the larger is $\alpha_{i}$. This implies that the manufacturer with high grade will have the opportunity to gain more profits. Constraint (2) expresses that the profit of each manufacturer should not be negative. Constraint (3) shows that all orders should be delivered to the enterprise before the deadline.

\subsection{Preliminary Results}

Theorem 1. There exists an optimal solution for the problem in which all of the following hold.

(i) There is no inserted idle time between orders processed on each machine.

(ii) The departure time of each shipment is the completion time of the last order included in the shipment.

(iii) Orders that are processed on the same machine and delivered in the same shipment are processed consecutively on that machine.

Corollary 2. If an order assignment schedule is given in the problem, the maximum lead time of the orders processing in manufacturer $i$ is independent of how the delivery shipments are formed, and it is equal to the sum of delivery time $t_{i}$ and completion time of the last order assigned to manufacturer $i$.

Theorem 3. There exists an optimal solution for the problem in which all the delivery shipments, except possibly one, at each manufacturer, are full. More precisely, if there are $h_{i}$ orders scheduled at manufacturer $i$, then $u b$ orders are delivered in $u$ full shipments and $v$ orders are delivered in a partial shipment:

$$
h_{i}=u b+v, \quad u \geq 0,0 \leq v<b .
$$

Corollary 4. If there are $h_{i}$ orders scheduled at manufacturer $i$ for a given order assignment schedule, $y_{i}$ shipments are the optimal number of batches from manufacturer $i$ to the enterprise. $y_{i}$ can be gotten from the following inequity:

$$
h_{i} \leq y_{i} b
$$

According to the above preliminary results, the problem can be formulated as follows.

Decision Variable. Consider the following. $x_{i j k}=1$ where order $j$ is assigned to the $k$ th machine of manufacturer $i$; 0 , otherwise.

$y_{i}$ is the number of shipments from manufacturer $i$.

Temporary Variable. $P_{i}$ is the profit of manufacturer $i$ :

$$
\begin{array}{ll}
\operatorname{Max} & \sum_{i \in M} \alpha_{i} P_{i} \\
\text { s.t. } & P_{i}=\sum_{j \in N} \sum_{k=1}^{r_{i}}\left(e_{j}-c_{i j}\right) x_{i j k}-f_{i} y_{i} \geq 0, \quad \forall i \in M \\
& \max _{k \in\left\{1, \ldots, r_{i}\right\}}\left(\sum_{j \in N} p_{i j} x_{i j k}\right)+t_{i} \leq D, \quad \forall i \in M \\
& \sum_{i \in M} \sum_{k=1}^{r_{i}} x_{i j k}=1, \quad \forall j \in N \\
& \sum_{j \in N} \sum_{k=1}^{r_{i}} x_{i j k} \leq b y_{i}, \quad \forall i \in M \\
& x_{i j k} \in\{0,1\}, \quad y_{i} \geq 0 \text { integer, } \\
& \forall k \in\left\{1, \ldots, r_{i}\right\} ; \quad \forall i \in M ; \forall j \in N .
\end{array}
$$

Constraint (8) is that the profit of each manufacturer should not be negative. Constraint (9) is to confirm that all orders should be delivered to the enterprise before due date. Constraint (10) confirms that all orders should be processed by a machine once. Constraint (11) is used to calculate the delivery batch.

Constraint (9) can also be described as follows:

$$
\sum_{j \in N} p_{i j} x_{i j k}+t_{i} \leq D, \quad \forall k \in\left\{1, \ldots, r_{i}\right\} ; \forall i \in M
$$

Thus, the model is converted into a mixed integer programming model. The mixed integer programming model can be solved by business software, such as ILOG-CPLEX and LINGO.

\section{Calculation Analysis}

The purpose of the numerical experiment is to verify the mixed integer programming model in a simulated instance. The model was coded in the Visual C++ 2005 and solved using the optimization package ILOG-CPLEX 12.2. The computation was executed using an Intel Core2 Duo CPU 2.2 $\mathrm{GHz}$ processor and 1.0 GB of RAM. 


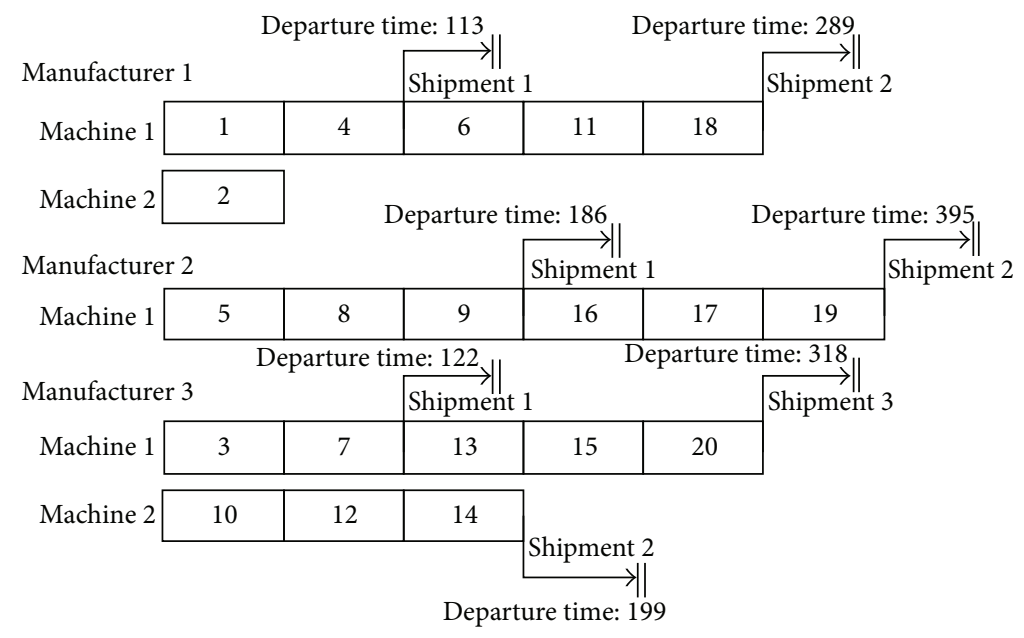

Figure 2: Optimal schedule for the instance.

We select a small instance with 3 manufacturers and 20 orders to verify the model. The parameters of the simulated instance are as follows:

number of manufacturers: $m=3$,

number of orders: $n=20$,

number of machines: $r_{1}=2, r_{2}=1, r_{3}=2$,

capacity limit: $b=3$,

due date: $D=1000$,

weight value: $\alpha_{i}=1, i=1,2,3$.

Parameters of orders including price of each order, processing time, and production cost for each order in each plant are listed in Table 1.

Parameters of distribution including delivery cost and delivery time are listed in Table 2.

The optimal objective value of the simulated instance is 1950.

The profit of manufacturer 1 is $P_{1}=569$.

The profit of manufacturer 2 is $P_{2}=650$.

The profit of manufacturer 3 is $P_{3}=731$.

The optimal solution of the simulated instance is listed in Table 3.

The detail information of the optimal solution can be shown in Figure 2.

\section{Conclusion}

In this paper, we study a static and deterministic integrated production-distribution scheduling problem with multiple independent manufacturers and develop a mixed integer programming model to maximize a weight sum of the profit of each manufacturer in the supply chain under the constraints that all orders should be finished before a common deadline and the profits of all manufacturer are not negative.
TABLE 1: Parameters of orders.

\begin{tabular}{lccccccc}
\hline Order & Price & \multicolumn{2}{c}{ Plant 1} & \multicolumn{2}{c}{ Plant 2} & \multicolumn{2}{c}{ Plant 3} \\
$j$ & $e_{j}$ & $p_{1 j}$ & $c_{1 j}$ & $p_{2 j}$ & $c_{2 j}$ & $p_{3 j}$ & $c_{3 j}$ \\
\hline 1 & 238 & 50 & 155 & 83 & 63 & 59 & 185 \\
2 & 163 & 79 & 75 & 81 & 86 & 96 & 75 \\
3 & 239 & 69 & 165 & 78 & 101 & 54 & 146 \\
4 & 223 & 63 & 72 & 66 & 183 & 75 & 60 \\
5 & 217 & 73 & 170 & 70 & 153 & 82 & 162 \\
6 & 222 & 55 & 176 & 77 & 179 & 93 & 108 \\
7 & 188 & 75 & 64 & 75 & 68 & 68 & 52 \\
8 & 217 & 91 & 171 & 53 & 55 & 76 & 82 \\
9 & 249 & 71 & 86 & 63 & 60 & 92 & 65 \\
10 & 158 & 75 & 58 & 87 & 191 & 75 & 56 \\
11 & 235 & 54 & 63 & 81 & 136 & 79 & 115 \\
12 & 242 & 97 & 169 & 77 & 127 & 62 & 188 \\
13 & 243 & 66 & 120 & 77 & 156 & 64 & 163 \\
14 & 212 & 73 & 67 & 76 & 109 & 62 & 199 \\
15 & 239 & 100 & 112 & 63 & 169 & 58 & 137 \\
16 & 204 & 94 & 128 & 66 & 150 & 88 & 106 \\
17 & 182 & 79 & 92 & 77 & 67 & 78 & 174 \\
18 & 158 & 67 & 183 & 91 & 200 & 98 & 116 \\
19 & 218 & 78 & 110 & 66 & 169 & 97 & 183 \\
20 & 153 & 72 & 171 & 75 & 62 & 74 & 116 \\
\hline & & & & & & &
\end{tabular}

TABLE 2: Parameters of distribution.

\begin{tabular}{ccccccc}
\hline \multicolumn{2}{c}{ Plant 1 } & \multicolumn{2}{c}{ Plant 2 } & \multicolumn{2}{c}{ Plant 3 } \\
$f_{1}$ & $t_{1}$ & $f_{2}$ & $t_{2}$ & $f_{3}$ & $t_{3}$ \\
\hline 151 & 295 & 121 & 318 & 142 & 365 \\
\hline
\end{tabular}

The problem can be solved efficiently when the number of plants, the number of orders, and the value of shipment limit are not big by using CPLEX. However, the time CPLEX required to solve the problem will increase exponentially 
TABLE 3: Optimal solution of the instance.

\begin{tabular}{lccc}
\hline Plant & Machine & $y_{i}$ & Orders \\
\hline 1 & 1 & 2 & $1,4,6,11,18$ \\
& 2 & 2 & 2 \\
\hline 2 & 1 & $2,8,9,16,17,19$ \\
3 & 1 & 3 & $3,7,13,15,20$ \\
& 2 & & $10,12,14$ \\
\hline
\end{tabular}

with the number of plants, the number of orders, and the value of shipment limit. Therefore, heuristic algorithm will be a valuable approach to solve the problem, which is worth studying in the future. In addition, in our model, we assumed that no vehicle routing is allowed among the manufacturers. Vehicle routing method is an efficient way for manufacturers to decrease the delivery cost, and it deserves future research.

\section{Conflict of Interests}

The authors declare that there is no conflict of interests regarding the publication of this paper.

\section{References}

[1] Z.-L. Chen, "Integrated production and outbound distribution scheduling: review and extensions," Operations Research, vol. 58, no. 1, pp. 130-148, 2010.

[2] N. G. Hall and C. N. Potts, "Supply chain scheduling: batching and delivery," Operations Research, vol. 51, no. 4, pp. 566-584, 2003.

[3] N. G. Hall and C. N. Potts, "The coordination of scheduling and batch deliveries," Annals of Operations Research, vol. 135, pp. 4164, 2005.

[4] G. Pundoor and Z.-L. Chen, "Scheduling a productiondistribution system to optimize the tradeoff between delivery tardiness and distribution cost," Naval Research Logistics, vol. 52, no. 6, pp. 571-589, 2005.

[5] Z.-L. Chen and G. L. Vairaktarakis, "Integrated scheduling of production and distribution operations," Management Science, vol. 51, no. 4, pp. 614-628, 2005.

[6] Z.-L. Chen and G. Pundoor, "Integrated order scheduling and packing," Production and Operations Management, vol. 18, no. 6, pp. 672-692, 2009.

[7] J. Dong, A. Zhang, Y. Chen, and Q. Yang, "Approximation algorithms for two-machine open shop scheduling with batch and delivery coordination," Theoretical Computer Science, vol. 491, pp. 94-102, 2013.

[8] C. A. Ullrich, "Integrated machine scheduling and vehicle routing with time windows," European Journal of Operational Research, vol. 227, no. 1, pp. 152-165, 2013.

[9] C. Low, C.-M. Chang, R.-K. Li, and C.-L. Huang, "Coordination of production scheduling and delivery problems with heterogeneous fleet," International Journal of Production Economics, vol. 153, pp. 138-148, 2014.

[10] Z.-L. Chen and G. Pundoor, "Order assignment and scheduling in a supply chain," Operations Research, vol. 54, no. 3, pp. 555$572,2006$.
[11] D. K. Jiang and B. Li, "Supply chain scheduling based on hybrid taboo search algorithm," Chinese Journal of Mechanical Engineering, vol. 47, no. 20, pp. 53-59, 2011.

[12] D. K. Jiang and B. Li, "Multi-plant supply chain scheduling with parallel machines based on taboo search algorithm," China Mechanical Engineering, vol. 23, no. 6, pp. 688-693, 2012.

[13] D. K. Jiang and B. Li, "Order assignment and scheduling with parallel machines," Chinese Journal of Management, vol. 10, pp. 919-924, 2013.

[14] H. S. Jagdev and J. Browne, "The extended enterprise- a context for manufacturing," Production Planning and Control, vol. 9, no. 3, pp. 216-229, 1998.

[15] R. Kawtummachai and N. Van Hop, "Order allocation in a multiple-supplier environment," International Journal of Production Economics, vol. 93-94, pp. 231-238, 2005.

[16] X. T. Qi, "Order splitting with multiple capacitated suppliers," European Journal of Operational Research, vol. 178, no. 2, pp. 421-432, 2007.

[17] E. A. Demirtas and Ö. Üstün, "An integrated multiobjective decision making process for supplier selection and order allocation," Omega, vol. 36, no. 1, pp. 76-90, 2008. 


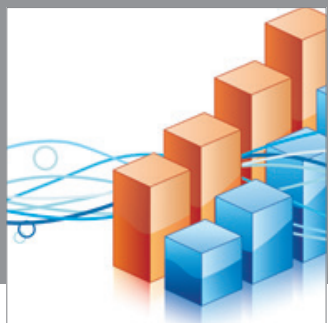

Advances in

Operations Research

mansans

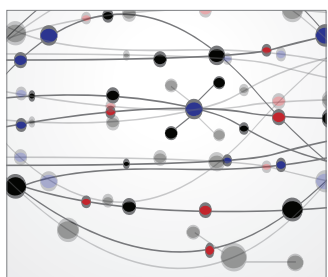

The Scientific World Journal
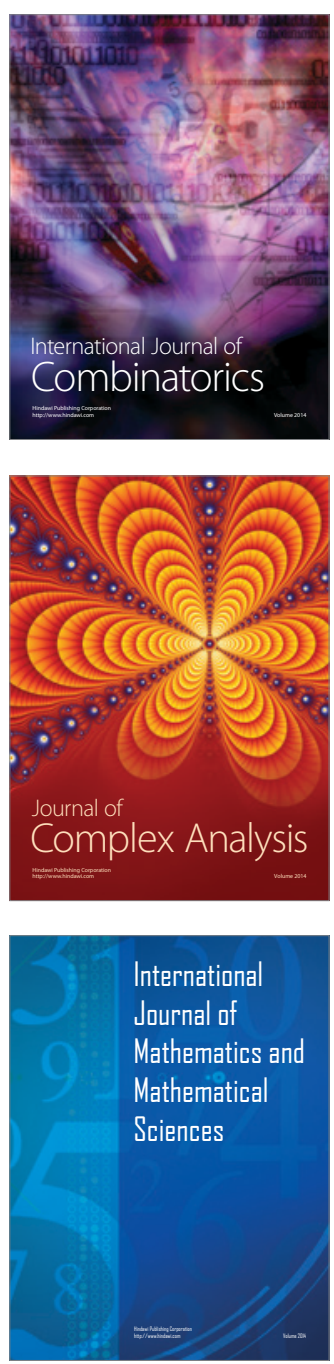
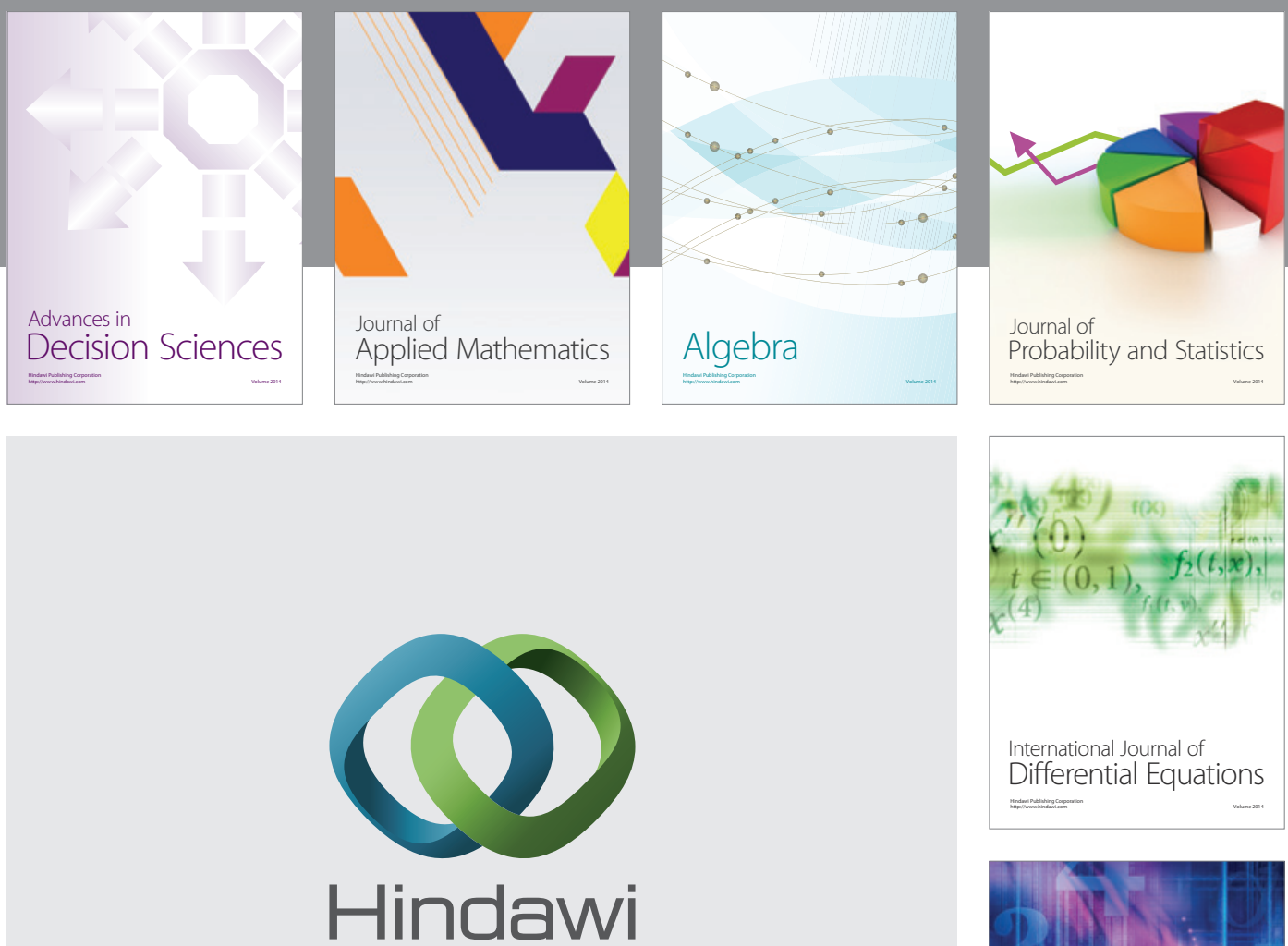

Submit your manuscripts at http://www.hindawi.com
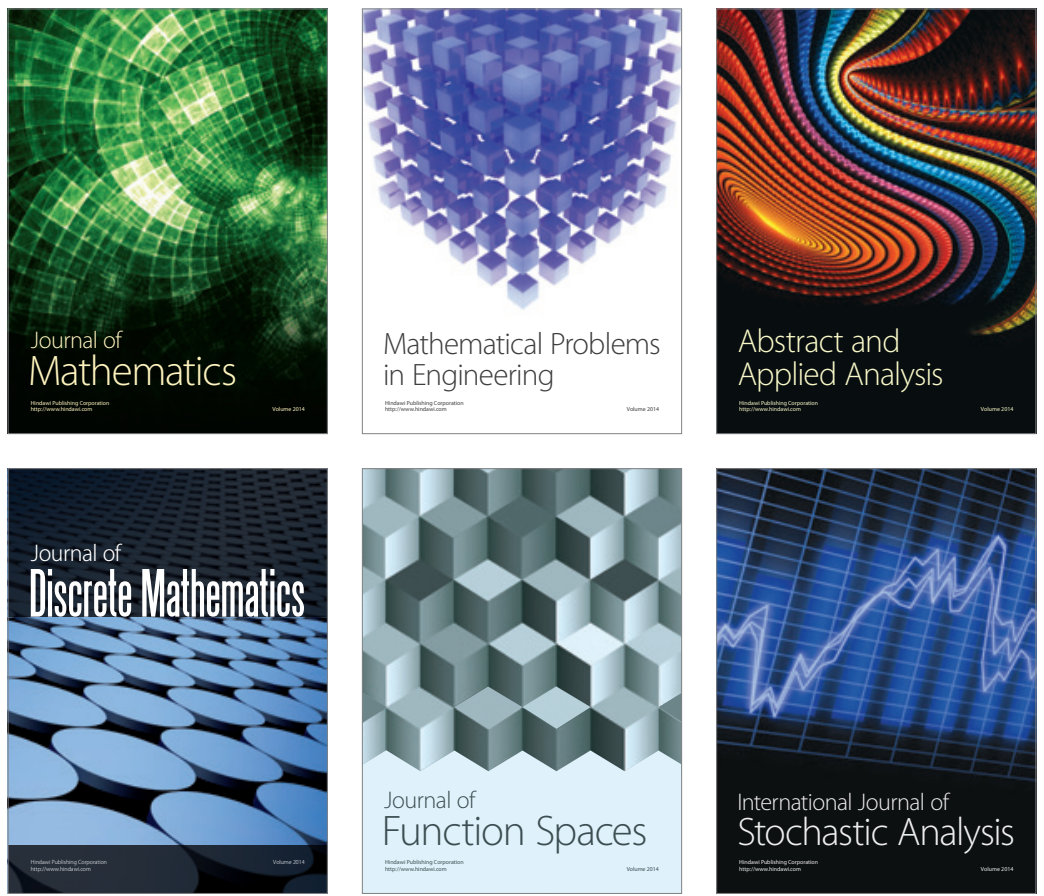

Journal of

Function Spaces

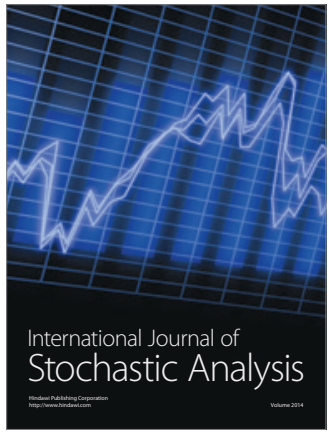

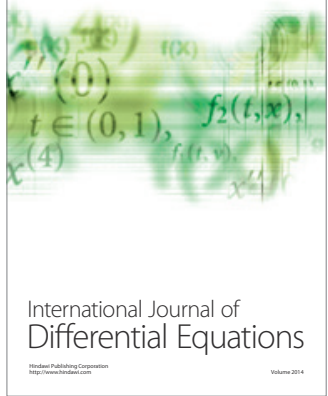
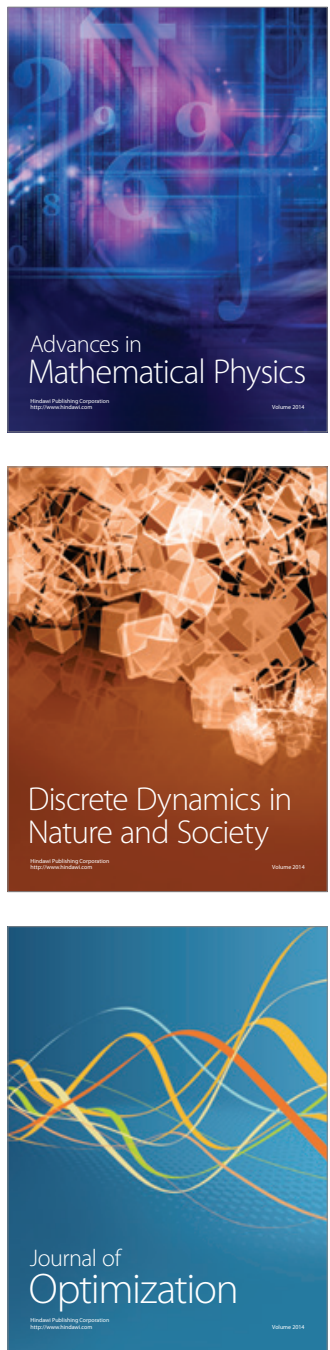